\title{
The research of the generalized tonic-clonic seizure epilepsy by resting state fMRI
}

\author{
Ming $\mathrm{Ke}^{1}$, Xiaoxin Duan $^{1}$, FanZhang ${ }^{1}$ \& Xiaoping Yang ${ }^{2}$ \\ ${ }^{1}$ College of Computer and Communication, Lanzhou University of Technology, Lanzhou, Gansu, \\ 730050, China
}

${ }^{2}$ Department of Imaging Diagnosis, Lanzhou General Hospital of Lanzhou Military Command, Lanzhou, Gansu, 730050, China.

Keywords: epilepsy, fMRI, Pearson correlation, network attributes, synchronicity

\begin{abstract}
In this study, we investigated the human brain functional network of patients with generalized tonic and clonic seizure (GTCS) epilepsy by using the resting-state functional magnetic resonance imaging (fMRI) and complex network theory. Adjacency matrices of the networks of GTCS group and normal control group were constructed by the method of Pearson correlation coefficient. A intra-group analysis was performed to get a statistical map of each group. And it was contrasted the attributes of brain network from two aspects of the "small world" property and network efficiency. Furthermore, a inter-group analysis was conducted to investigate differences between GTCS group and normal control group. Our results showed the "small world" property and network efficiency of GTCS group were decreased compared to the controls. In addition, the patients exhibited increased correlation coefficient mainly concentrated in the thalamus and the lentiform nucleus, while the decreased correlation coefficient was mainly distributed in the precuneus and frontal lobe, the inferior frontal gyrus, the inferior parietal gyrus, the superior parietal gyrus. The result indicated that information transmission ability of brain function network in patients with GTCS is impaired. And it also suggested that the method of Pearson correlation coefficient can detect synchronization change of blood-oxygen levels in the brain so that achieved the goal of positioning detection of epileptic activity.
\end{abstract}

\section{Introduction}

Epilepsy is a common neurological disorder characterized by abnormal hyper-synchronization of neural activity. GTCS usually have no obvious abnormity in brain structure. But as the emergence of the fMRI technology and a variety of algorithms, we have a certain understanding of the pathogenesis of the GTCS. For example, the structure, function and metabolism of the thalamus and thalamus cortical circuits are abnormal[1]. The resting-state fMRI need no task design in advance. People just need to be calm when scanned. It is perfect for the study of patients who have the obstacles of executive ability such as epilepsy and schizophrenia. In recent years, the research of the pathogenesis of GTCS by the resting-state fMRI technology has become a hot spot. Some scholars have done the research by using the fMRI techniques such as the independent component analysis (ICA)[2], amplitude of low frequency fluctuation (ALFF)[3] and regional homogeneity (ReHo) etc. This research adopts the method of Pearson correlation coefficient to construct brain function network at resting-state. It is contrasted the attributes of brain network from two aspects of the "small world" property and network efficiency and then explored the pathophysiological mechanism of GTCS and the applications of the algorithm.

\section{Materials and Methods}

\section{Subjects}

The fMRI data of this study is from the department of imaging diagnosis, Lanzhou general hospital of Lanzhou military command. According to the clinical diagnosis, 15 cases of patients 
participate in the analysis. At the same time, 15 cases of normal volunteers who have no neurological disorder are as the control group. The two groups are all right-handed and the gender, age have no statistical difference. The study is approved by the medical ethics committee of Lanzhou general hospital of Lanzhou military command. All participants know the research purposes before scanned and sign the consent form. Detailed clinical data are shown in table 1.

Table 1 The clinical data of patients with GTCS and normal volunteer

\begin{tabular}{ccccccc}
\hline & $\begin{array}{c}\text { Mal } \\
\text { e }\end{array}$ & $\begin{array}{c}\text { Femal } \\
\text { e }\end{array}$ & $\begin{array}{c}\text { Age } \\
\text { (year } \\
\text { ) }\end{array}$ & $\begin{array}{c}\text { Average } \\
\text { age (year) }\end{array}$ & $\begin{array}{c}\text { Duratio } \\
\mathrm{n} \\
\text { (year) }\end{array}$ & $\begin{array}{c}\text { Average } \\
\text { duration } \\
\text { (year) }\end{array}$ \\
\hline GTCS & 9 & 6 & $14 \sim 49$ & $24.17 \pm 6.4$ & $1 \sim 18$ & $5.72 \pm 6.68$ \\
$\begin{array}{c}\text { Volunte } \\
\text { er }\end{array}$ & 8 & 7 & $22 \sim 38$ & 8 & - & - \\
& & & & $\begin{array}{c}26.12 \pm 6.1 \\
2\end{array}$ & & \\
\hline
\end{tabular}

\section{Data Acquisition}

All subjects' resting-state data of the whole brain were collected by the Siemens $3.0 \mathrm{~T}$ mri scanners. Subjects were asked to keep head still, close eyes, relax and without specific thinking activity as far as possible when lie in magnetic resonance. At the same time plug their ears and dim the indoor lights. The parameters are as follows: recycling time $(T R)=2000 \mathrm{~ms}$, echo time $(\mathrm{TE})=30 \mathrm{~ms}$, filed of view $(\mathrm{FOV})=240 \mathrm{~mm}$, matrix $=64 \times 64$, slice thickness $=3.8 \mathrm{~mm}$, gap $=0.38 \mathrm{~mm}$. Collect 200 time points in 400 seconds.

\section{Data Preprocessing}

Preprocess fMRI images by DPARSF software: (1)Convert DICOM data format into NIFTI.(2) Remove the top-ten time points of each participant to achieve stable signal. (3)Make the data collected in different time corrected to the same time point. (4)Eliminate the participants whose translation of head is greater than $1 \mathrm{~mm}$ and rotation is greater than 1.5 degrees. (5)Normalize the image to the standard space. (6) Smooth by the gaussian kernel function. (7)Remove the linear trend. 8Low frequency filter. Filter the frequency of $0.01-0.08 \mathrm{~Hz}$ to remove the effects of breathing, heart rate and other physiological noise.

\section{Functional Connectivity}

Calculate the function connection between the regions of interesting based on the method of Pearson correlation coefficient. Firstly, remove the covariate. Then divide the whole brain into 90 regions of interesting based on brain atlas Anatomical Automatic Labeling. Thirdly, Extract the time sequence of the region of interesting. Finally calculate the correlation coefficient $C(r, s)$ by the time sequence of the region of interesting $r$ and ${ }^{S}$.Computation formula is as follow:

$$
c(r, s)=\frac{\sum_{i=1}^{n}[r(i)-\bar{R}] \bullet[s(i)-\bar{S}]}{\sqrt{\sum_{i=1}^{n}[r(i)-\bar{R}]^{2} \bullet \sum_{i=1}^{n}[s(i)-\bar{S}]^{2}}}
$$

$r(i), s(i)$ are the time sequence of the region of interesting $r$ and $S$ respectively. $\bar{R}, \bar{S}$ are the average of $r(i), s(i)$ respectively. that is

$$
\begin{gathered}
\bar{R}=\frac{1}{n} \sum_{i=1}^{n} r(i) \\
\bar{S}=\frac{1}{n} \sum_{i=1}^{n} s(i)
\end{gathered}
$$

There is functional connection relations between $r$ and $s$ when $C(r, s)$ is greater than the selected threshold $\mathrm{T}$. The corresponding element of the adjacency matrix which is used to describe the network structure is 1 , otherwise is 0 . In order to meet the actual characteristics of the human 
brain, the value of T must meet the demand that the average degree $<k>$ of network can't less than the natural logarithm of node number $(\ln (N)$ )of the network.

\section{The Analysis of Network Properties}

The study use the random networks as a benchmark to quantify the "small world" property. The network belongs to the category of "small world" network If the actual network has the larger cluster coefficient and shortest path length relative to the random network. Then the cluster

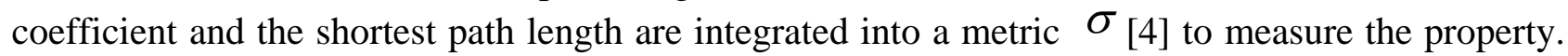
The network has the "small world" property when $\sigma>1$, and the bigger $\sigma$ is, the stronger the "small world" property of the network. Namely

$$
\begin{gathered}
\gamma=C_{\text {real }} / C_{\text {random }} \gg 1 \\
\lambda=L_{\text {real }} / L_{\text {random }} \approx 1 \\
\sigma=\gamma / \lambda
\end{gathered}
$$

The $C_{\text {real }}$ and $C_{\text {random }}$ represent the cluster coefficient of actual networks and random networks respectively; $L_{\text {real }}$ and $L_{\text {random }}$ represent shortest path length of actual networks and random networks respectively.

Then the study adopt the local efficiency (Eloc) and global efficiency (Eglob) to measure properties of brain networks further because that the cluster coefficient only considered the direct connection between neighbor nodes and the shortest path length is usually operated in a connected graph. The nodes which are not connected in the network will lead to the value of the shortest path length between two nodes infinite.

The local efficiency of any node is as follow:

$$
E(i)=\frac{1}{N_{G_{i}}\left(N_{G_{i}}-1\right)} \sum_{j \neq k \in G_{i}} \frac{1}{l_{j, k}}
$$

Global efficiency is:

$$
E_{g l o b}=\frac{1}{N(N-1)} \sum_{i, j \in V, i \neq j} \frac{1}{1_{i j}}
$$

$G_{i}$ refers to subgraph consisted of neighbor notes of ${ }^{i},{ }^{l_{k k}}$ represents the shortest path length between note ${ }^{j}$ and note $k$.

\section{The Results and Discussion}

According to the research in recent years, the number of edges in the network will be reduced and close to the sparse network as the threshold increases. The weak connection and the noise will be ruled out. But if the threshold is too high, the isolated nodes in network will be increased significantly. The small world property will not be correctly estimated when the average degree $<k>$ of network less than the natural logarithm of node number. Figure 1 shows the average degree of network under different threshold. From the picture you can see that the average degree of the network is slightly greater than the natural logarithm of node number when $T=0.5$. So we choose $T=0.5$ to analyze the properties of resting-state brain function network.

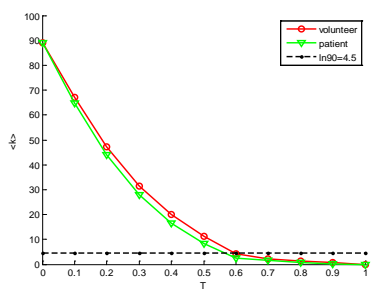

Figure 1 average degree of network under different threshold 
Table 2 shows the parameters results of brain networks at the resting state of GTCS group and normal control group. It can be seen that the values of $\sigma$ are 1.6601 and 2.0685 on the condition of $\mathrm{T}=0.5$, which shows the function network all have the "small world" property, but compared with normal control group, the property of GTCS group decreased. Figure 2 shows further that the clustering coefficient, global efficiency and local efficiency of GTCS group are lower than the normal control group. While the shortest path length is higher. The higher the efficiency of network, the faster the rate of information transmission between nodes of the network. The property of "small world" and efficiency of network decline in the GTCS group suggest that the information transmission ability is damaged.

The study also shows the increased, decreased and the unchanged connection between the GTCS group and the normal control group from stereoscopic, coronal, sagittal and axial image further. It's not hard to find the brain areas which the connection increased mainly concentrated in the occipital lobe and thalamus, the decreased areas mainly concentrated in the parietal lobe and frontal lobe (figure 3). Among which, the specific links are shown in table 3.

Table 2 The result of parameters of GTCS group and normal control group

\begin{tabular}{cccccccc}
\hline & $\mathrm{C}$ & $\mathrm{L}$ & $\gamma$ & $\lambda$ & $\sigma$ & Eloc & Eglob \\
\hline Volunte & 0.48 & 2.454 & 2.2263 & 1.0763 & 2.0685 & 0.4103 & 0.4454 \\
er & 0.438 & 2 & 2.0339 & 1.2251 & 1.6601 & 0.3585 & 0.4151 \\
Patient & 5 & 2.793 & - & - & - & - & - \\
Rando & 0.215 & 5 & & & & & \\
m & 6 & 2.280 & & & & & \\
& & 2 & & & & &
\end{tabular}

Note: Patient represents GTCS group; Volunteer represents the normal control group; Random represents random network; $C$ represents cluster coefficient; $L$ represents the shortest path length; $\gamma=C_{\text {real }} / C_{\text {random }} ; \lambda=L_{\text {real }} / L_{\text {random }} ; \sigma=\gamma / \lambda$; Eloc represents local efficiency; Eglob represents global efficiency.

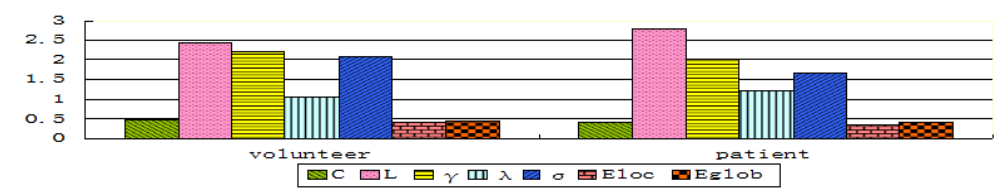

Figure 2 The network properties of GTCS group (patient) compared with normal control group (volunteer)

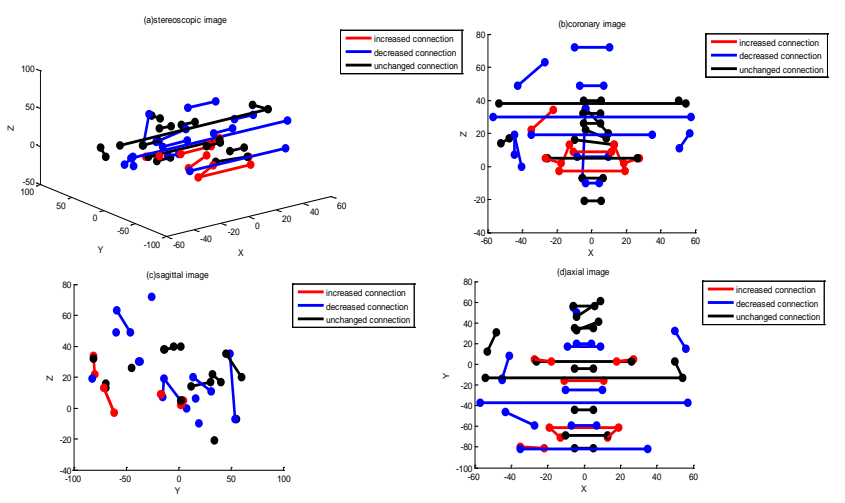

Figure3 The increased, decreased and the unchanged connection between the GTCS group and the normal control group

note: (a) stereoscopic image, (b) coronary image, (c)sagittal image, (d) axial image; Red, blue and black lines represent the brain connections that the correlation coefficient is increased, decreased and unchanged respectively. 
Table 3 Compared with normal control group,the brain areas of GTCS that Pearson Correlation Coefficient changes significantly

\begin{tabular}{cc|cc}
\hline \multicolumn{2}{c|}{$\begin{array}{c}\text { Pearson Correlation Coefficient } \\
\text { increased }\end{array}$} & \multicolumn{2}{c}{$\begin{array}{c}\text { Pearson Correlation Coefficient } \\
\text { decreased }\end{array}$} \\
\hline AALbrain areas & AALbrain areas & AALbrain areas & AALbrain areas \\
\hline Calcarine_L & Lingual_L & Frontal_Inf_Oper_ & Frontal_Inf_Tri_R \\
Calcarine_R & Lingual_R & R & factory_R \\
Lingual_L & Lingual_R & factory_L & Frontal_Mid_Orb_L \\
Occipital_Sup_L & Occipital_Mid_ & Frontal_Sup_Media & Insula_L \\
Putamen_L & L & l_LRolandic_Oper_ & Heschl_L \\
Putamen_R & Pallidum_L & L & Parietal_Sup_L \\
Thalamus_L & Pallidum_R & Rolandic_Oper_L & Occipital_Mid_R \\
& Thalamus_R & Parietal_Inf_L & SupraMarginal_R \\
& & Occipital_Mid_L & Precuneus_R \\
& & SupraMarginal_L & \\
& & Precuneus_L & \\
\hline
\end{tabular}

Pearson correlation coefficient reflects the time synchronization of BOLD signal and the strength of brain activity. The study shows that the Pearson correlation coefficient of thalamus of GTCS has the biggest change compared with normal control group, which indicates that the synchronization of neural activity is enhanced. In the past studies. Gotman[5] proposed that the neuron abnormal discharge of thalamus cortex is associated with large seizures of epilepsy. Thalamus and adjacent mesh structure are considered to be the initiation site of discharge. At the same time, Ciumas[6] reported that the structure and metabolism of thalamus of GTCS is abnormal, namely the volume of gray matter of the bilateral thalamus decreased. In addition, the Pearson correlation coefficient of lenticular nucleus and lenticular pallidum are also increased in this study. Luo[7]found that the function connections of basal ganglia of IGE are higher than normal, particularly the putamen, to a certain extent, provide the basis for the regulating role of basal ganglia in the study of IGE. $\mathrm{Li}$ [8]found the perfusion of thalamus and basal ganglia of GTCS decreased during the attack. The consistency of these results of neuroimaging studies illustrate that the anomaly of basal ganglia is associated with GTCS attack further. In this study, the synchronization enhancement of thalamus and basal ganglia may be influenced by the activity of GTCS epilepsy source, which prompts those brain regions are associated with the generation and propagation of epilepsy activities of GTCS.

In addition, our result also find the brain areas that the correlation coefficient decreased are mostly located in the default network, particularly the precuneus and frontal lobe, which indicates that the whole brain prolonged discharge of epilepsy patients may result in the default network damaged. The result is consistent with result of low frequency amplitude of the resting- state fMRI. Wang Maoxue[9] found that the ALFF of bilateral precuneus and medial prefrontal lobe are decreased which may be associated with impaired cognitive function of GTCS. The default network of brain is related with the extraction of episodic memory and the monitoring to the surrounding environment. Therefore, the result that synchronicity of default network reduced compared with ordinary people in this study can further explain why people with epilepsy existed for a long time disturbance of consciousness and verify the theory that the brain default networks are severely affected on the condition of serious disturbance of consciousness.

\section{Conclusions}

In this study, the "small world" property and network efficiency are decreased. The information transmission ability of brain network is damaged. In addition, the strengthen of Pearson correlation coefficient suggests that the brain regions are associated with the generation and propagation of epilepsy activities. The decreased value suggests that long-term repeated discharge of the whole 
brain may result in damage of default network. The results are consistent with the results obtained with other methods. The method of Pearson correlation coefficient has the ability to detect seizure activity of GTCS and can be used in clinical research of epilepsy effectively.

\section{Acknowledgments}

The work was partially supported by the National Science Foundation of China (61263074), and the Fundamental Research Funds for Gansu Universities.

\section{References}

[1] Zhang Z,Liao W,Chen H. Altered functional-structural coupling of large-scale brain networks in idiopathic generalized epilepsy[J].Brain,2011,(10):2912-2928

[2]Morgan VL,Gore JC,Abou-Khalil B.Cluster analysis delection of functional MRI activity in temporallobeepilepsy[J]. 2007(01)

[3]Zhang Zhiqiang,Lu Guangming,Zhong Yuan,Tan Qifu,Zhu Jianguo,Jing Li,Chen Zhili,Wang Zhongqiu,Shi Jixin,Zang Yufeng,Liu Yijun.Application of amplitude of low-frequency fluctuation to the temporal lobe epilepsy with bilateral hippocampal sclerosis:an fMRI study[J].National Medical Journal of China,2008(23)

[4]Humphries M D,Gurney K,Prescott T J.The brainstem reticular formation is a small world, not scale-free, network[J].Proc R Soc Lond BBiol Sci,2006,273:503-511

[5]Gotman J,Grova C,Bagshaw A,et a1.Generalized epileptic discharges show thalamocortical activation and suspension of the default state of the brain[J].Proc Natl Acad Sei USA,2005,102(42):15236-15240

[6] Ciumas C.Savic I-Structural changes in patients with primarygeneralized tonic and clonic seizures[J].Neurology,2006,67(4):683-686

[7] Luo C,Li Q,Xia Y,et a1.Resting state basal gang1ia network jn idiopathic generalized epilepsy[J].Hum Brain Mapp,2011.Apr21.[Epub ahead of print]

[8] Li Q,Luo C,Yang T,et a1.EEG-fMRl study on the interictal and ictal generalized spike-wave discharges in patients with childhood absence epilepsy[J].Epilepsy Res,2009,87(2-3):160-168

[9]Wang Maoxue,Zhang Zhiqiang,Wang Zhengge,Shen Lianfang,Lu guangming.Study of amplitude of low frequency with generalized tonic clonic fluctuation in interictal epilepsy seizures based on fMRI[J].China medical imaging technology,2011,27(10):1985-1989 